\title{
Steady flow of horizontal double-sided symmetric thin liquid films
}

\author{
Joseph G. Abdulahad, Shaker M. Rasheed *, Hakima K. Ahmed \\ Department of Mathematics, Faculty of Science, University of Zakho, Kurdistan Region, Iraq
}

\section{A R T I C LE I N F O}

\section{Article history:}

Received 10 June 2016

Received in revised form

8 September 2016

Accepted 12 September 2016

\section{Keywords:}

Steady flow

Thin liquid film

Navier-Stokes equation

\begin{abstract}
A B S T R A C T
In this study, The steady flow of and within horizontal thin liquid symmetric double-sided film is considered. The nonlinear differential equations that govern such flow are derived from the Navier-Stokes equation. The solution curves are obtained numerically by using MATLAB software for two nondimensional cases. Numerical method and MATLAB are used to obtain the solutions and plot the curves for the result nonlinear differential equation and for number of fluids.
\end{abstract}

(C) 2016 The Authors. Published by IASE. This is an open access article under the CC BY-NC-ND license (http://creativecommons.org/licenses/by-nc-nd/4.0/).

\section{Introduction}

The problem of the dynamic system has been widely studied. This paper focused on a class of dynamic system whose surface tension has a significant mechanical effect on the system as a whole and no solid boundaries presented. In several areas in science and engineering a considerable attention has been devoted to study the flow within thin liquid films. Abdulahad (1989), discussed the nature of the boundary condition at the free surface of the liquid when, the liquid is in the form of a moving thin film. The effect of inertia on the rupture process of thin liquid film is studied by Erneux and Davis (1993), and assumed that the film is thin enough to neglect the gravity effect and the effect of the Van der Waals potential is considered because of thin thickness. He used integral method to study the inertia effect on rupture process of thin liquid film and numerical method to solve nonlinear evolution equation in order to study the rupture process. In Abdulahad and Derbaz (2014), the thinning process of an inclined thin liquid film over a solid boundary with an inclination angle $\alpha$ to the horizontal in gravity driven flow and some of the theoretical aspects of the instability development in an inclined thin liquid films on a solid surface are studied. A mathematical model is constructed by Abdulahad (2010) to describe a two dimensional flow for an inclined films with an inclination angle $\alpha$ to the horizontal that drainages under the action of gravity. Abdulahad and Derbaz (2014), is considered the

\footnotetext{
* Corresponding Author.

Email Address: shaker.rasheed.mathematics@gmail.com (S. Rasheed)

http://dx.doi.org/10.21833/ijaas.2016.08.015

2313-626X/C) 2016 The Authors. Published by IASE.

This is an open access article under the CC BY-NC-ND license

(http://creativecommons.org/licenses/by-nc-nd/4.0/)
}

thinning process of an inclined thin liquid films on a solid surface for unsteady flow where the gravity and other forces such as viscous and surface tension forces have a significant effect on the flow by using similarity solution. In Abdulahad and Hamad (2015), it has been considered the unsteady flow within a horizontal double-sided symmetric thin liquid film with negligible inertia. The similarity and perturbation methods are used to obtain a nonlinear differential equation that governs such flow for unsteady state in dimensionless form. A partial differential equation is derived in (Faraidun, 2007) which describe the evolution of the interface shape subject to surface tension, viscous forces, plus van der Waals attractions. The stability and dynamics of a thin liquid films flowing down on an inclined plane by using integral approximation are investigated by (Ismael, 2014) and the strong non-linear evolution equations are derived by the integral approximation with a specified velocity profile.

In this paper, the steady flows of and within thin liquid symmetric double-sided film is considered. The differential equation that governs such flow is considered and the solutions curves are obtained and it is shown graphically.

\section{Formulation and governing equations}

The flow under investigation has been modeled as a steady, two dimensional flow of incompressible fluid within a horizontal double-sided symmetric thin liquid film; the coordinates $\mathrm{x}$ and $\mathrm{z}$ designate the directions parallel and normal to the flow respectively as shown in Fig. 1. Two free surfaces are located at $\mathrm{z}= \pm \mathrm{h}(\mathrm{x})$, where $\mathrm{z}$ is the transverse coordinate, $x$ is the axis of symmetry. For thin liquid films we assume that $\frac{\partial \mathrm{h}}{\partial \mathrm{x}} \ll 1$ for all $\mathrm{x}$. Let $\mathrm{u}(\mathrm{x}, \mathrm{z})$ and $\mathrm{w}(\mathrm{x}, \mathrm{z})$ denote the corresponding velocity 
filed of the fluid, where $\mathrm{u}$ and $\mathrm{w}$ are the velocity component in $\mathrm{x}$ and $\mathrm{z}$ directions respectively. For two dimensional motions of the liquid, we determine the velocity and pressure distribution as follow:

In differential form, the Navier-Stokes equations without external forces in $\mathrm{x}$ and $\mathrm{z}$ directions for steady incompressible flow are given by:

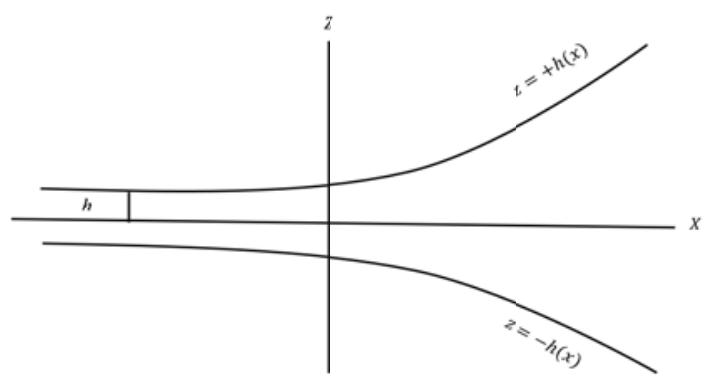

Fig. 1: Cross- section of a symmetric film

$$
\rho\left[u \frac{\partial u}{\partial x}+w \frac{\partial u}{\partial z}\right]=-\frac{\partial P}{\partial x}+\mu\left[\frac{\partial^{2} u}{\partial x^{2}}+\frac{\partial^{2} u}{\partial z^{2}}\right],
$$

and

$$
\rho\left[\mathrm{u} \frac{\partial \mathrm{w}}{\partial \mathrm{x}}+\mathrm{w} \frac{\partial \mathrm{w}}{\partial \mathrm{z}}\right]=-\frac{\partial \mathrm{P}}{\partial \mathrm{z}}+\mu\left[\frac{\partial^{2} \mathrm{w}}{\partial \mathrm{x}^{2}}+\frac{\partial^{2} \mathrm{w}}{\partial \mathrm{z}^{2}}\right]
$$

The velocity distribution for the flow is given by:

$$
\left.\begin{array}{c}
u=u(x) \\
v=0 \\
w=-z \frac{\partial u}{\partial x} \\
p=p(x)
\end{array}\right\}
$$

which satisfies the incompressibility condition,

$$
\frac{\partial u}{\partial x}+\frac{\partial w}{\partial z}=0
$$

since the slope $\frac{\partial \mathrm{h}}{\partial \mathrm{x}}$ is so small for thin liquid films, the distribution satisfies the stress condition at the surface of film $\mathrm{z}=\mathrm{h}(\mathrm{x})$, which are:

\section{Shear stress condition:}

$$
\tau=\mu\left(\frac{\partial u}{\partial x}\right)_{s}=0 \text {, }
$$

where $\mu$ is the viscosity of liquid $\frac{\partial u}{\partial x}$ is the velocity gradient and the subscribe $s$ denotes the values at the surface of the film.

\section{Normal stress condition:}

$$
\mathrm{T}_{\mathrm{zz}}=\left(-\mathrm{p}+2 \mu \frac{\partial \mathrm{w}}{\partial \mathrm{z}}\right)_{\mathrm{s}} \text {. }
$$

Now from the continuity equation (2.1.4), we have:

$$
\frac{\partial \mathrm{w}}{\partial \mathrm{z}}=-\frac{\partial \mathrm{u}}{\partial \mathrm{x}}
$$

substituting it into equation (2.1.4), equation (2.1.6) gives:

$$
\mathrm{T}_{\mathrm{zz}}=\left(-\mathrm{p}-2 \mu \frac{\partial \mathrm{u}}{\partial \mathrm{x}}\right)_{\mathrm{s}}
$$

The curvature of the liquid film is given by:

$$
\mathrm{k}=\frac{\partial^{2} \mathrm{~h}}{\partial \mathrm{x}^{2}}\left(1+\left(\frac{\partial \mathrm{h}}{\partial \mathrm{x}}\right)^{2}\right)^{-3 / 2}
$$

Since, $\frac{\partial \mathrm{h}}{\partial \mathrm{x}} \ll 1$, then $\left(\frac{\partial \mathrm{h}}{\partial \mathrm{x}}\right)^{2}$ can be neglected and equation (2.1.9) reduces to give:

$$
\mathrm{k}=\frac{\partial^{2} \mathrm{~h}}{\partial \mathrm{x}^{2}}
$$

also, on the surface of the liquid film with surface tensiono, the normal component of stress is given by,

$$
\mathrm{T}_{\mathrm{zz}}=\sigma \frac{\partial^{2} \mathrm{~h}}{\partial \mathrm{x}^{2}}
$$

From equations (2.1.8) and (2.1.10) and on the surface of the film, we have:

$$
\mathrm{p}=-2 \mu \frac{\partial \mathrm{u}}{\partial \mathrm{x}}-\sigma \frac{\partial^{2} \mathrm{~h}}{\partial \mathrm{x}^{2}}
$$

which holds everywhere. Notice that $\mathrm{p}, \mathrm{u}$ and $\mathrm{h}$ are functions of $x$ only. Differentiate equation (2.1.12) with respect to $x$, we get:

$$
\frac{\partial p}{\partial x}=-2 \mu \frac{\partial^{2} u}{\partial x^{2}}-\sigma \frac{\partial^{3} h}{\partial x^{3}}
$$

from the velocity distributions, the longitudinal equation of motion (2.1.1) for steady flow reduces to give:

$$
\rho\left(u \frac{\partial u}{\partial x}\right)=-\frac{\partial p}{\partial x}+\mu \frac{\partial^{2} u}{\partial x^{2}} .
$$

Equations (2.1.12) and (2.1.14), give:

$$
\rho\left(u \frac{\partial u}{\partial x}\right)=\sigma \frac{\partial^{3} h}{\partial x^{3}}+3 \mu \frac{\partial^{2} u}{\partial x^{2}} .
$$

From the velocity distributions, the transverse equation of motion (2.1.2), gives,

$$
\frac{\partial p}{\partial z}=z\left(\rho u \frac{\partial^{2} u}{\partial x^{2}}-\rho\left(\frac{\partial u}{\partial x}\right)^{2}-\mu \frac{\partial^{3} u}{\partial x^{3}}\right)
$$

Integrating equation (2.1.17) with respect to $\mathrm{z}$, we get:

$$
\mathrm{p}=\frac{\mathrm{z}^{2}}{2}\left(\rho \mathrm{u} \frac{\partial^{2} \mathrm{u}}{\partial \mathrm{x}^{2}}-\rho\left(\frac{\partial \mathrm{u}}{\partial \mathrm{x}}\right)^{2}-\mu \frac{\partial^{3} \mathrm{u}}{\partial \mathrm{x}^{3}}\right)+\mathrm{g}(\mathrm{x}),
$$

which can be written in the form:

$$
\mathrm{p}=\mathrm{p}(\mathrm{x})+\mathrm{o}\left(\mathrm{z}^{2}\right),|\mathrm{z}| \leq \mathrm{h}
$$


This is relevant only to higher order approximation.

Since $\mathrm{z}=\mathrm{h}(\mathrm{x})$ is a free surface of the liquid film, then the conservation of the mass across the film thickness of the film is therefore given by:

$$
\mathrm{uh}=\mathrm{Q},
$$

where $\mathrm{Q}$ is any constant.

Thus, equations (2.1.16) and (2.1.19) are the governing equations of and within the liquid film.

\section{Flows with negligible inertia}

The governing equation (2.1.16) with negligible inertia reduces to give:

$$
\frac{\partial^{3} \mathrm{~h}}{\partial \mathrm{x}^{3}}+\frac{3 \mu}{\sigma} \frac{\partial^{2} \mathrm{u}}{\partial \mathrm{x}^{2}}=0
$$

From equation (2.2.1), the only material constant that is relevant is therefore:

$$
\mathrm{V}=\frac{\sigma}{3 \mu}
$$

We can determine the value of the parameter $\mathrm{V}$ for some liquids as shown in the following table.

Every solution of equations (2.1.16) and (2.1.19) must be assessed with respect to full equations given in the previous section. In particular, for infinitesimal perturbations as a uniform film and from Table 1, the analysis shows that inertia can never be neglected for mercury; can only marginally be neglected in water and carbon tetrachloride; and can always be neglected in thin films of glycerin, linseed oil and olive oil.

From equations (2.2.1) and (2.2.2), we have:

$$
\frac{\partial^{3} \mathrm{~h}}{\partial \mathrm{x}^{3}}+\frac{1}{\mathrm{~V}} \frac{\partial^{2} \mathrm{u}}{\partial \mathrm{x}^{2}}=0
$$

Integrating equation (2.2.3) with respect to $\mathrm{x}$ twice, we get:

$$
\frac{\partial \mathrm{h}}{\partial \mathrm{x}}+\frac{1}{\mathrm{~V}} \mathrm{u}=\mathrm{Ax}+\mathrm{B}
$$

\begin{tabular}{|c|c|c|c|c|}
\hline Liquid & $\begin{array}{l}\text { density } \\
\rho, / \mathrm{cm}^{3}\end{array}$ & $\begin{array}{l}\text { Surface tension } \\
\sigma, \mathrm{m} / \mathrm{sec}^{2}\end{array}$ & $\begin{array}{c}\text { viscosity } \\
\mu, \mathrm{g} / \mathrm{cm} \text {. sec }\end{array}$ & $\begin{array}{c}\text { Velocity } \\
\text { V, cm/sec }\end{array}$ \\
\hline Water & 0.998 & 72.97 & 0.0113 & 2152.5074 \\
\hline Mercury & 13.55 & 510.76 & 0.0115 & 10984.086 \\
\hline Glycerin & 1.26 & 62.75 & 14.9 & 1.4038 \\
\hline $\begin{array}{c}\text { Carbon } \\
\text { Tetrachloride }\end{array}$ & 1.59 & 26.27 & 0.00974 & 899.0418 \\
\hline Linseed oil & 0.94 & 33.57 & 0.4309 & 25.9698 \\
\hline Olive oil & 0.91 & 33.56 & 0.8379 & 13.3508 \\
\hline
\end{tabular}

where, A and Bare arbitrary constants and can be found from asymptotic or initial conditions.
From equation (2.1.19), equation (2.2.4) reduces to give:

$$
\frac{1}{v}\left(\frac{Q}{h}\right)+\frac{d h}{d x}=A x+B
$$

Equation (2.2.5) is related to lubrication theory, but in the absence of surface -active solutes, it seems to be a degenerate relationship; since the shear stress at the edge of the film is then zero, and this ensures that the velocity distribution across the film is uniform, not parabolic.

Now equation (2.2.5) gives the following two cases:

Case I:

If $A \neq 0$, we can write $A x+B=D x$ and thus equation (2.2.5), then becomes:

$\frac{1}{\mathrm{v}}\left(\frac{\mathrm{Q}}{\mathrm{h}}\right)+\frac{\mathrm{dh}}{\mathrm{dx}}=\mathrm{Dx}$.

Case II:

If $A=0$, then equation (2.2.5), gives:

$\frac{1}{v}\left(\frac{Q}{h}\right)+\frac{d h}{d x}=B$

\section{Non-dimensional analysis}

For non-trivial solution of equation (2.2.6), we define the following non- dimensional parameters for case I as follows:

$$
\left.\begin{array}{l}
x=\frac{Q^{\frac{1}{3}}}{v^{\frac{1}{3}} D^{\frac{2}{3}}} \eta \\
h(x)=\frac{Q^{\frac{2}{3}}}{v^{\frac{2}{3}} D^{\frac{1}{3}}} f(\eta)
\end{array}\right\},
$$

and equation (2.2.6), reduces to give:

$$
f(\eta) \frac{d f}{d \eta}-\eta f(\eta)=-1
$$

The following analysis follows from the locus of term of the function of $f_{c}(\eta)$ which is the critical solution of equation (2.3.9). Note that we use ODE45 and plot commands in MATLAB to solve (2.3.9) and all the curves in this paper.

The locus of points at which $\frac{\mathrm{df}}{\mathrm{d} \eta}=0$ may be written from equation (2.3.9), as:

$$
f(\eta)=\frac{1}{\eta}
$$

Some of the solution curves are obtained for equation (2.3.9) in $(\eta, f(\eta))$-plane. Within the class of the solutions there is a critical solution shown in Fig. 
2 which divided the region of the definition into two sub regions. The behavior is as follows:

$$
\mathrm{f}_{\mathrm{c}}(\eta) \sim \frac{1}{2} \eta^{2} \text { as } \eta \rightarrow-\infty
$$

and

$$
\mathrm{f}_{\mathrm{c}}(\eta) \sim \frac{1}{\eta} \text { as } \eta \rightarrow \infty
$$

Now as $\rightarrow-\infty$, all the films have constant curvature, and $f_{c}(\eta)$ may be related to the supply of fluid to thin films from a plateau border (a region of large curvature).

Note that no solution intersects $f_{c}(\eta)$, and all the solution curves which lie above the critical solution $\mathrm{f}_{\mathrm{c}}(\eta)$, and have the asymptotic behavior:

$$
f(\eta)=\frac{1}{2} \eta^{2} \text { as } \eta \rightarrow \pm \infty
$$

The behavior (2.3.13) corresponds to the behavior:

$$
\mathrm{h}(\mathrm{x})=\frac{1}{2} \mathrm{kx}^{2} \text { as } \mathrm{x} \rightarrow \pm \infty \text {. }
$$

In $(\mathrm{x}, \mathrm{h}(\mathrm{x}))$-plane, where $\mathrm{k}$ is arbitrary constant which labels each solution, so that all of these solutions describe the transition from a film of uniform thickness to one of constant curvature. All the solution curves which lie below the critical solution $f_{c}(\eta)$ in Fig. 2 have the asymptotic behavior:

$$
\mathrm{f}_{\mathrm{c}}(\eta) \sim \frac{1}{2} \eta^{2} \text { as } \eta \rightarrow \infty \text {, }
$$

but $\mathrm{f}_{\mathrm{c}}\left(\eta_{0}\right)=0$ with $\mathrm{u}_{0} \rightarrow \infty$.

The behavior of (2.3.18) corresponds to:

$$
\mathrm{h}(\mathrm{x}) \sim \frac{1}{2} \mathrm{kx}^{2} \text { as } \mathrm{x} \rightarrow-\infty \text {, }
$$

but $\mathrm{h}(\mathrm{x})=0$ for all values of $\mathrm{x}$ with $\mathrm{u}\left(\mathrm{x}_{0}\right)=\infty$, and this represents a film which terminates in a sink of fluid at $\mathrm{x}=\mathrm{x}_{0}$, where the thin film approximation breaks down.

In $(\mathrm{x}, \mathrm{h}(\mathrm{x}))$-plane Fig. 3 shown the thickness of the film for different liquids namely: Mercury, Carbon Tetrachloride, Water, Glycerin, Linseed oil and Olive oil. The comparison of the thickness of films for some liquids is shown in Fig. 4 and 5.

For non-trivial solution of equation (2.2.7), we define the following non- dimensional parameters for case II as follows:

$$
\left.\begin{array}{l}
x=\frac{Q}{V^{2}} \eta \\
h(x)=\frac{Q}{V B} f(\eta)
\end{array}\right\},
$$

and thus equation (2.3.7), reduces to give

$$
\frac{1}{\mathrm{f}(\eta)}+\frac{\mathrm{df}}{\mathrm{d} \eta}=1
$$

or

$$
\left(1+\frac{1}{f(\eta)-1}\right) \frac{d f}{d \eta}=1
$$

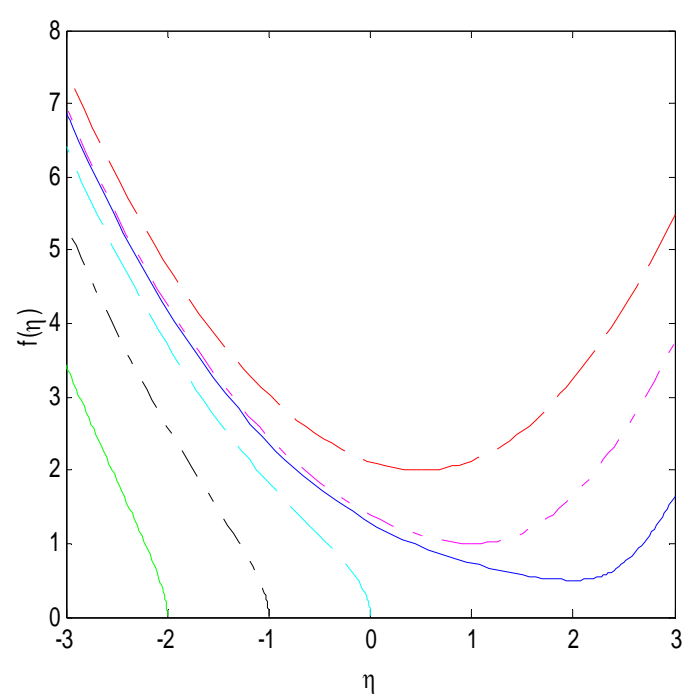

Fig. 2: Solution curves of equation (2.3.9) in $(\eta, f(\eta))$ plane.
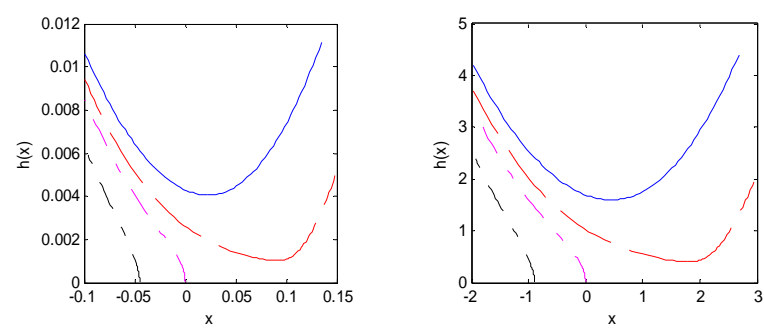

Mercury

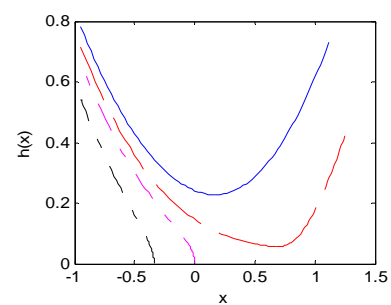

Linseed oil

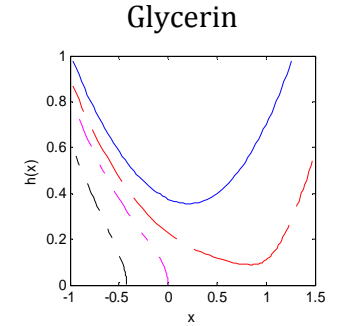

Olive oil

Fig. 3: Solution curves of equation (2.3.9) in $(\mathrm{x}, \mathrm{h}(\mathrm{x}))$ plane, for different liquids.

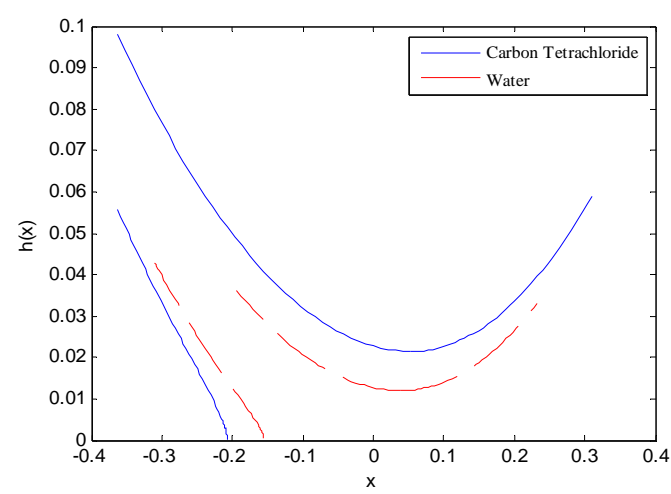

Fig. 4: Comparison between the film thickness of carbon tetrachloride and water

Again we use ODE45 command in MATLAB to solve (2.3.18).

Equation (2.3.19) is the governing equation for case II. Integrating equation (2.3.19) with respect to $\eta$, we get: 


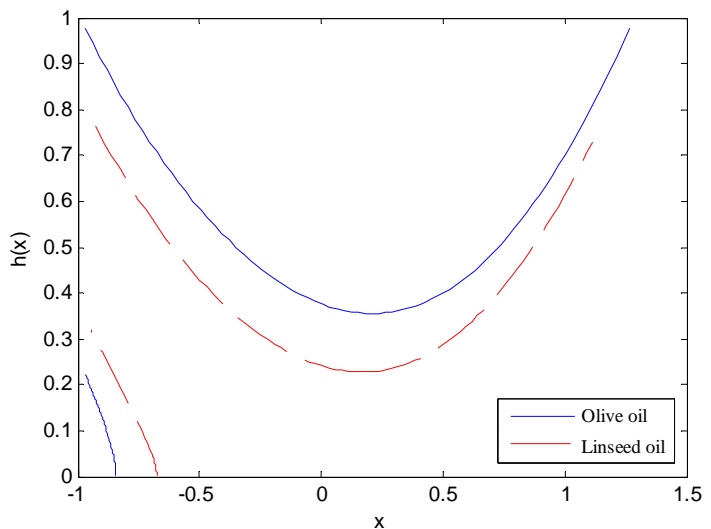

Fig. 5: Comparison between the film thickness of olive oil and linseed oil

$$
f(\eta)+\ln (f(\eta)-1)=\eta-\ln \left(k_{1}\right)
$$

or

$$
f(\eta)+\ln \left(k_{1}(f(\eta)-1)\right)=\eta
$$

where $\mathrm{k}_{1}(\mathrm{f}(\eta)-1)>0$.

Some of the solution curves are obtained from equation (2.3.20) in $(\eta, f(\eta))$-plane as shown in Fig. 6. The locus of points for which $\frac{d f}{d \eta}=0$ and from equation (2.3.18) gives,

$$
f(\eta)=1
$$

This is also represented the critical solution $f_{c}(\eta)$ of equation (2.3.21).All the solution curves which lie above the critical solution $\mathrm{f}_{\mathrm{c}}(\eta)$ have the asymptotic behavior,

$$
f(\eta) \sim \eta \text { as } \eta \rightarrow \infty
$$

which corresponds to the behavior:

$$
\mathrm{h}(\mathrm{x}) \sim \mathrm{Ax} \text { as } \mathrm{x} \rightarrow \infty,
$$

in $(\mathrm{x}, \mathrm{h}(\mathrm{x}))$ - plane, where $\mathrm{A}$ is arbitrary constant which labels each solution. Now the remaining solution curves which lie below the critical solution have the asymptotic behavior:

$$
\mathrm{f}(\eta) \sim 1 \text { as } \eta \rightarrow-\infty \text {, }
$$

but

$$
f\left(\eta_{0}\right)=0 \text { as } u_{0} \rightarrow \infty
$$

Again the solutions represent a film which terminates in a sink of fluid where the thin film approximation breaks down.

In $(\mathrm{x}, \mathrm{h}(\mathrm{x}))$-plane Fig. 7 shown the thickness of the film for different liquids namely: Mercury, Carbon Tetrachloride, Water, Glycerin, Linseed oil and Olive oil. The comparison of the thickness of films for some liquids is shown in Fig. 8 and 9.

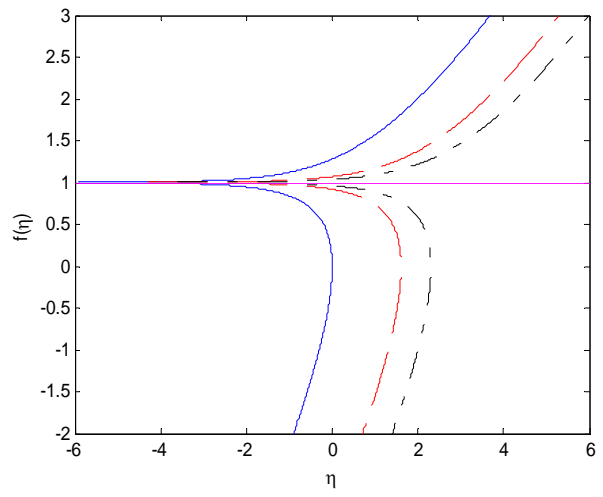

Fig. 6: Solution curves of equation (2.3.19) in $(\eta, f(\eta))$ plane for different value of $\mathrm{k}_{1}$.

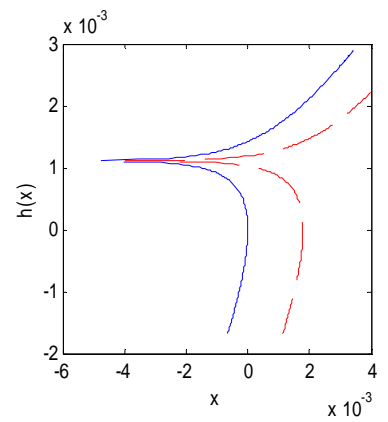

Carbon tetrachloride

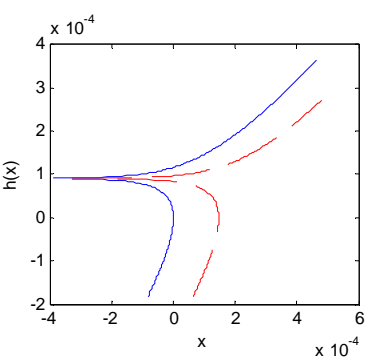

Mercury

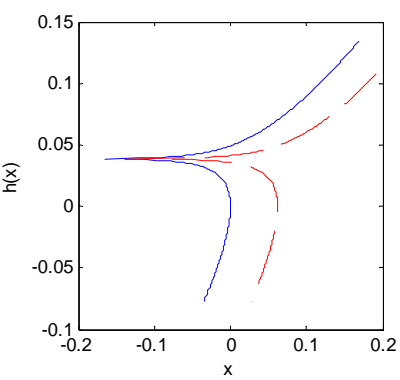

Linseed oil

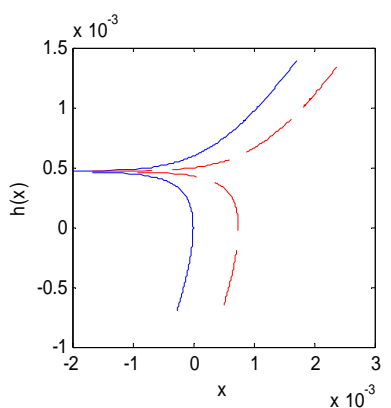

water

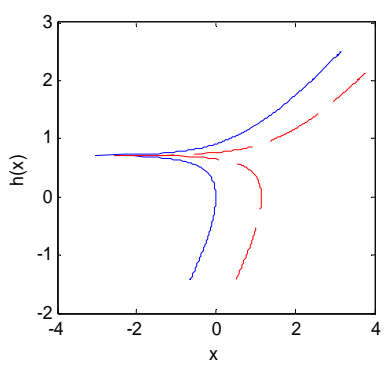

Glycerin

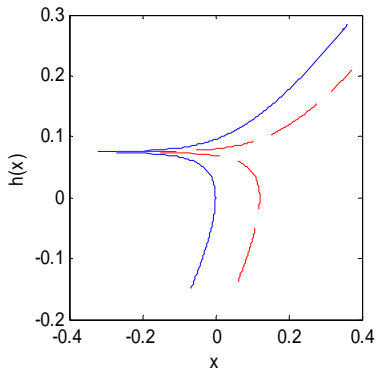

Olive oil
Fig. 7: Solution curves of equation (2.3.19) in $(\mathrm{x}, \mathrm{h}(\mathrm{x}))$

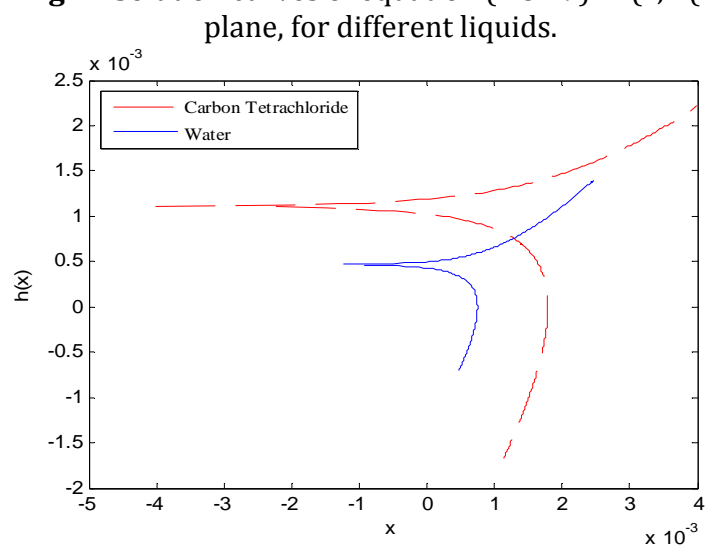

Fig. 8: Comparison between the film thickness of carbon tetrachloride and water 


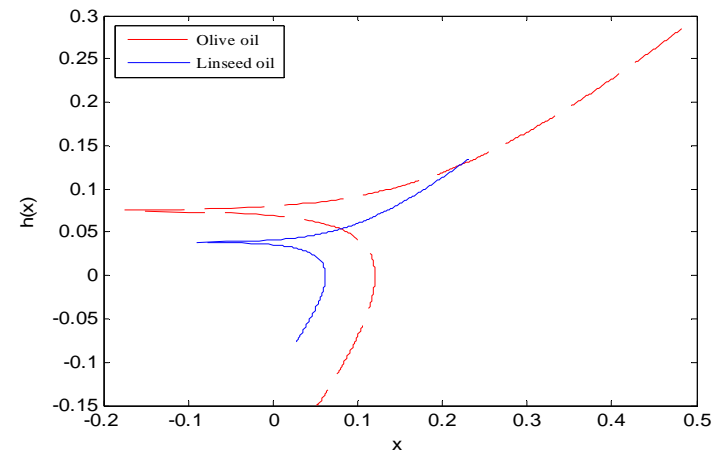

Fig. 9: Comparison between the film thickness of Olive oil and Linseed oil.

\section{Conclusion}

Two dimensional flow of incompressible fluid within a horizontal double-sided symmetric thin liquid film with negligible inertia is considered. The nonlinear differential equation that governs such flow for steady flow is obtained for two cases. The non-dimensional form of the non-linear differential equation for the first case is obtained and solved numerically by using Range Kutta method (ODE45).

Fig. 2 shows some of the solution curves of this case in $(\eta, f)$ - plane, where the region of definition of the solution divided into two sub regions by a critical solution $f_{c}(\eta)$ which may be related to the supply of liquid to a thin film from the border. The curves which lie above the critical solution are asymptotic to $f(\eta)=\frac{1}{2} \eta^{2}$ as $\eta$ tends to $\pm \infty$. The curve which lie below the critical solution have asymptotic behavior to $f(\eta)=\frac{1}{2} \eta^{2}$ as $\eta$ tends to $\infty$, but $f\left(\eta_{0}\right)=0$ as $\mathrm{u}_{0} \rightarrow \infty$ and then it terminates to a sink where the velocity $\mathrm{u}$ becomes very large when the thickness of the film $\mathrm{h}$ is tends to zero as shown in Fig. 3. Fig. 3 shows the film thickness for different liquids, namely Water, Carbon Tetrachloride, Mercury, Glycerin, Linseed oil and Olive oil in ( $\mathrm{x}, \mathrm{h}(\mathrm{x}))$-plane. For the second case, we found that the non-dimensional form of the non-linear differential equation has an exact solution which is obtained analytically and some of the solution curves are plotted by using Mat lab program and shown in Fig. 6. The region of the solution curves of this case in $(\eta, f(\eta))$-plane is again divided into two sub regions by a critical solution given by $f_{c}(\eta)=1$. The curves above the critical solution are asymptotic to $f(\eta)=\eta$ as $\eta$ tends to $\infty$. The curves below the critical solution are asymptotic to $f(\eta)=1$ as $\eta$ tends to $-\infty$ and with $f\left(\eta_{0}\right)=0$ as $\mathrm{u}_{0} \rightarrow \infty$ and again these solutions represent a film which terminates in a sink of fluid where the film approximation breaks down as shown in Fig. 6 . Furthermore, the film thickness for different liquids, namely Water, Carbon Tetrachloride, Mercury, Glycerin, Linseed oil and Olive oil in $(\mathrm{x}, \mathrm{h}(\mathrm{x}))$-plane are shown in Fig. 3 and 7 for the two cases. The comparison of the thickness of films shows that the thickness of Water is less than that of Carbon Tetrachloride and this may be because, the surface tension of water is greater than that of carbon tetrachloride as shown in Fig. 4 and 8. Also, the thickness of Olive oil is more than that of Linseed oil for the viscosity of linseed oil is less than that of Olive oil as it is shown in Fig. 5 and 9. Whereas, for infinitesimal perturbations as a uniform film the analysis shows that inertia can never be neglected in mercury because of the large value of the velocity as given in Table 1.

\section{References}

Abdulaha JG and Derbaz SA (2014). The drainage of thin liquid films on an inclined solid surface, IOSR Journal of Mathematics, 10(2): 73-80.

Abdulahad JG (1989). Liquid surface conditions. Ph.D thesis, University of Essex, England.

Abdulahad JG (2010). Flow of Soap Films on Inclined Plane. Rafidain Journal of Computer Sciences and Mathematics, 7(2): 67-78.

Abdulahad JG and Derbaz SA (2014). Steady Flow of Thin Liquid Films on an Inclined Solid surface. IOSR Journal of Engineering, 4(4): 41-46.

Abdulahad JG and Hamad IS (2015). Unsteady Flow in a Double-Sided Symmetric Thin Liquid Films. IOSR Journal of Mathematics, 11(3): 38-48.

Erneux T and Davis SH (1993). Nonlinear rupture of free films. Physics of Fluids A: Fluid Dynamics (1989-1993), 5(5): 1117-1122.

Faraidun KHS (2007). Curtain Coating flow of an Inclined Thin Liquid Films. AL-Rafidain Journal of Computer Sciences and Mathematics, 4(2): 99111.

Ismael HF (2014). Linear Stability of Thin Liquid Films flows down on an Inclined Plane using Integral Approximation. AL-Rafidain Journal of Computer Sciences and Mathematics, 11(2): 1324. 